\title{
Altered Amino Acid Metabolic Patterns in the Plasma of Rat Models with Adenovirus Infection
}

\author{
Man Jeong Paik, ${ }^{a}$ Wooyoung Shim, ${ }^{a}$ Seung Min Moon, Yeon Mi Kim, ${ }^{\dagger}$ Dong Wan Kim, ${ }^{\dagger}$ \\ Kyoung-Rae Kim, ${ }^{\S}$ Sun A Kim, Jeom Soon Shim, Sangdun Choi, and Gwang Lee ${ }^{\ddagger}$ \\ Department of Molecular Science and Technology, Ajou University, Suwon 443-721, Korea. *E-mail: glee@ajou.ac.kr \\ 'Department of Microbiology, College of Natural Sciences, Changwon National University, Changwon 641-773, Korea \\ ${ }^{\S}$ Biometabolite Analysis Laboratory, College of Pharmacy, Sungkyunkwan University, Suwon 440-746, Korea \\ Institute for Medical Science, Ajou University School of Medicine, Suwon 443-749, Korea \\ Received October 8, 2010, Accepted March 19, 2011
}

\begin{abstract}
The presence of replication-competent adenovirus (RCA) subpopulations in adenoviral vector products raises a variety of safety issues for development of therapies based on gene therapy. To analyze the differing effects of adenoviral vector and RCA in vivo, we examined alterations in amino acids (AAs) using rat plasma following injection of $\beta$-galactosidase expressing recombinant adenovirus (designated rAdLacZ) or RCA. Plasma AAs were examined by gas chromatography-mass spectrometry. A total of 16 AAs were positively measured. In the rAdLacZ group compared to the control group, the level of aspartic acid was significantly increased (Student's t-test), while the level of glutamic acid was significantly reduced. Additionally, in the RCA group compared to the control group, the level of four AAs, valine, leucine, and isoleucine as branched-chain amino acids, and proline were significantly increased, whereas the levels of three AAs, glycine, threonine, and glutamic acid were significantly reduced. Altered plasma free AA metabolic patterns in rAdLacZ and RCA groups, compared with the control group, may explain the disturbance of AA metabolism related to viral infection.
\end{abstract}

Key Words : Adenoviral vector, Amino acid, Replication-competent adenovirus, Star pattern recognition

\section{Introduction}

Replication-deficient adenoviral vectors have been widely used as gene delivery systems that should have the ability to transfer exogenous genes directly to a variety of mammalian cells and tissues for gene therapy. ${ }^{1-6}$ The adenovirus is particularly attractive as a vector since it can be produced in high titer $\left[10^{10}-10^{11}\right.$ plaque forming units $\left.(\mathrm{pfu}) / \mathrm{mL}\right]$ and generate highly purified recombinant virus, which efficiently infects target cells, and does not require host cell replication. ${ }^{7}$ However, there have been a few critical evaluations on the safety of recombinant adenoviral vectors. ${ }^{8-10}$ It is important to consider the consequences of recombinant adenovirus or RCA infection in vivo. In contrast to our knowledge, little is known regarding changes in metabolites by direct in vivo gene transfer using adenoviral vectors, including replicationcompetent adenovirus (RCA) for the safety of human gene therapy trials.

In our recent reports, altered metabolic patterns of free fatty acids (FFAs) ${ }^{11}$ and polyamines (PAs) ${ }^{12}$ as important biochemical indicators of pathological condition ${ }^{13-16}$ were studied in rat plasma after injection with two adenoviruses, $\beta$-galactosidase expressing recombinant adenovirus (rAdLacZ) and RCA. FFA levels in the plasma of rAdLacZ and RCA rats were significantly different compared with those of control rats. ${ }^{11}$ The star patterns of the rAdLacZ and RCA groups were readily distinguishable from each other. In addition, altered PA levels were significantly different in $t$ he two adenovirus infection groups compared with the normal group. ${ }^{12}$ These results suggested that adenoviral infection may be monitored with metabolite as an abnormal state. Especially, endogenous metabolism of PAs are highly correlated with amino acids (AAs) metabolism, since PAs are synthesized biologically from AA, i.e., ornithine, methionine, arginine, lysine. And AAs are also well known as energy source by depletion of carbohydrate or fatty acid amounts. Therefore, we extended our study to the metabolic patterns of AAs.

The stimulation of innate immune response by adenoviruses can result in inflammation of non-targeted tissues following metabolic changes that modify protein and induce AAs. However, few studies have been investigated on the establishment of a system with which to monitor the effects of adenoviral infection on AA associated metabolic changes. Maranga et al. reported on the association of cellular metabolism with adenoviral in vitro. ${ }^{17}$ They found that adenoviral infection induced a higher consumption of most essential AAs, including principal nutrients, i.e., glucose, and oxygen for viral propagation. This suggested that adenoviral infection may affect AA metabolism in vivo. However, the relation between viral infection and AA metabolism has not been studied. 


\section{Experimental}

Cell Cultures. Human embryonic kidney cells (HEK 293 cells) and human alveolar basal epithelial cells (A549 cells) were obtained from the Korean Cell Line Bank (Seoul, South Korea). All cell lines were cultured in Dulbecco's modified Eagle's medium supplemented with $10 \%$ fetal bovine serum (Bio Whittaker) and grown at $37^{\circ} \mathrm{C}$ in a humidified atmosphere containing $5 \% \mathrm{CO}_{2}$.

Adenoviruses and Viral Assay Methods. Two kinds of adenoviruses were used throughout this study, as described previously. ${ }^{12}$ Briefly, the recombinant adenovirus, rAdLacZ, which expresses the Escherichia coli lacZ gene coding $\beta$ galactosidase, and RCA (adenovirus type 5) were obtained from the American Type Culture Collection (ATCC VR1516). The infection titer of RCA on HEK 293 cells was $7 \times$ $10^{10}$ NAS Infection Units (NIU)/mL as a reference, and the frozen stock was confirmed to have retained its titer. $\mathrm{rAdLacZ}$ was purified using an Adeno-XIM Virus Purification Kit (BD Biosciences), and then concentrated using Centricon TM-50 centrifugal filter units (Millipore, Billerica, MA, USA) and titrated in HEK 293 cells using an Adeno-XTM Rapid Titer Kit (BD Biosciences) according to supplier instructions.

Polymerase Chain Reaction (PCR). Adenoviral genomic DNA samples were extracted from purified rAdLacZ and RCA following addition of proteinase K, phenol-chloroform extraction, and ethanol precipitation. To detect contamination of RCA in rAdLacZ by PCR, the following E1 specific primer pair was used: 5'-TCCGGTCCTTCTAACACA-3' and 5'-CAGGCTCGTTAAGCAAGT-3'. PCR was carried out with an initial denaturation step at $94{ }^{\circ} \mathrm{C}$ for $5 \mathrm{~min}$, followed by 30 cycles, including a denaturation step $\left(94{ }^{\circ} \mathrm{C}\right.$ for $1 \mathrm{~min})$, an annealing step $\left(55^{\circ} \mathrm{C}\right.$ for $\left.1 \mathrm{~min}\right)$, an extension step $\left(72{ }^{\circ} \mathrm{C}\right.$ for $\left.1 \mathrm{~min}\right)$, and a final extension step at $72{ }^{\circ} \mathrm{C}$ for $5 \mathrm{~min}$. To analyze 293 cells derived from genomic DNA, a pregnancy-specific glycoprotein (PSG) gene was used as a target. The following primer pairs were used: 5'-CTCATGCCTGCCTCTTTCACT-3' and 5'-AGAGCCATCCACACAATGTGC-3', as previously reported. ${ }^{8}$ Samples were amplified with initial denaturation at $94{ }^{\circ} \mathrm{C}$ for $5 \mathrm{~min}$, followed by 30 cycles of denaturation $\left(94^{\circ} \mathrm{C}\right.$ for $\left.1 \mathrm{~min}\right)$, an annealing step $\left(57^{\circ} \mathrm{C}\right.$ for $\left.1 \mathrm{~min}\right)$, an extension step $\left(72^{\circ} \mathrm{C}\right.$ for $\left.1 \mathrm{~min}\right)$, and a final extension at $72{ }^{\circ} \mathrm{C}$ for $5 \mathrm{~min}$. All amplified DNA samples were analyzed by gel electrophoresis in $1 \%$ agarose gel in tris-acetate-ethylenediaminetetra-acetate (TAE) buffer, and DNA fragments were visualized by ethidium bromide staining. Las-1000 was used for detection of DNA bands, with Las-1000Plus software (Fujifilm).

Animal Studies and Plasma Specimens. This study was approved by the Institutional Animal Care and Use Committee (AMC-17). Sprague-Dawley rats (250-300 g) were used in this study and two types of adenovirus were used: rAdLacZ, which expresses the Escherichia coli lacZ gene coding b-galactosidase, and RCA (adenovirus type 5) were obtained from the American Type Culture Collection (ATCC VR-1516), as in a previous report. ${ }^{12}$ Briefly, prior to viral injection, animals were anesthetized by chloral hydrate solution (Fluka) and infected with $0.1 \mathrm{~mL}$ total volume of phosphate-buffered saline (PBS) diluted to contain $1 \times 10^{10}$ pfu into a tail vein. Plasma samples were collected from three normal control rats, four rats infected with rAdLacZ virus, and three rats infected with RCA virus at $72 \mathrm{~h}$ after injection. All samples were immediately stored at $-70{ }^{\circ} \mathrm{C}$ until analysis. In order to exclude variation in diet, since dietary supplementation is associated with the level of AAs in plasma, we used control rats that were supplied the same food. Sample preparation for AAs in rat plasma, AAs were converted into their ethoxycarbonyl (EOC)/tert-butyldimethylsilyl (TBDMS) derivatives, as in our previous reports. ${ }^{18}$

Chemicals and Reagents. The 16 AA standards, 3,4-dimethoxybenzoic acid as an internal standard (IS), and ethyl chloroformate (ECF) were obtained from Sigma-Aldrich (St. Louis). $N$-Methyl- $N$-(tert-butyldimethylsilyl)trifluoroacetamide (MTBSTFA) was purchased from Pierce (Rockford). All other chemicals of analytical grade were used.

Preparation of Standard Solutions. The stock solution of each AA was made up at $10 \mu \mathrm{g} / \mu \mathrm{L}$ in $0.1 \mathrm{M} \mathrm{HCl}$. Standard working solutions of 0.01 and $0.5 \mu \mathrm{g} / \mu \mathrm{L}$ were then prepared by diluting each stock solution with $0.1 \mathrm{M} \mathrm{HCl}$, as in the previous report. ${ }^{18}$ Briefly, the IS stock solution, prepared by dissolving at $10.0 \mu \mathrm{g} / \mu \mathrm{L}$ in methanol, was used to make an IS working solution of $0.05 \mu \mathrm{g} / \mu \mathrm{L}$ in methanol. Mixed calibration samples were prepared at five concentrations ranging from 0.05 to $5.0 \mu \mathrm{g} / \mathrm{mL}$ by mixing appropriate aliquots of each working solution. All standard solutions were stored at $4{ }^{\circ} \mathrm{C}$ until analysis.

Sample Preparation for AAs in Rat Plasma. AAs were converted into their EOC)/TBDMS derivatives following a previous method. ${ }^{18}$ Briefly, $0.5 \mathrm{~mL}$ of acetonitrile to remove protein was added to $0.1 \mathrm{~mL}$ of rat plasma (three normal, four rAdLacZ, and three RCA samples) containing $0.5 \mu \mathrm{g}$ of IS. The mixture was diluted with $0.5 \mathrm{~mL}$ of distilled water and centrifuged $(15,000 \mathrm{rpm}, 15 \mathrm{~min})$, and the supernatant layer was adjusted to a $\mathrm{pH}$ of 12 with $5.0 \mathrm{M} \mathrm{NaOH}$. A twophase EOC reaction was then performed in one step by vortex-mixing (5 min) an aqueous phase with ECF $(20 \mu \mathrm{L})$ present in the dichloromethane phase $(1.0 \mathrm{~mL})$.

The reaction mixture was acidified $(\mathrm{pH} \leq 2.0)$ using concentrated sulfuric acid. Then it was extracted with diethyl ether $(3.0 \mathrm{~mL})$ and ethyl acetate $(2.0 \mathrm{~mL})$ sequentially, and the combined extracts were evaporated to dryness under a gentle nitrogen stream $\left(40{ }^{\circ} \mathrm{C}\right)$. The residue was reacted $\left(60{ }^{\circ} \mathrm{C}\right.$ for $\left.30 \mathrm{~min}\right)$ with MTBSTFA $(20 \mu \mathrm{L})$ in toluene $(20$ $\mu \mathrm{L}$ ) for GC-MS analysis in selected ion monitoring (SIM).

Gas Chromatography-Mass Spectrometry. GC-MS analyses in both scan and SIM modes were performed using an Agilent 6890 gas chromatograph, interfaced with an Agilent 5975B mass-selective detector (70 eV, electron impact mode) installed with an Ultra-2 (5\% phenyl-95\% methylpolysiloxane bonded phase; $25 \mathrm{~m} \times 0.20 \mathrm{~mm}$ i.d., $0.11 \mu \mathrm{m}$ film thickness) cross-linked capillary column (Agilent Technologies), as in our previous reports. ${ }^{12,18}$

Star Symbol Plotting. The concentration of each AA in 
normal, rAdLacZ, and RCA plasma samples was determined based on calibration curves. The AA levels in each sample were normalized to the corresponding mean in the control group. Subsequently, each normalized value was plotted as a line radiating from a common central point, and the far ends of the lines were joined together to produce star patterns using MS Excel, as described elsewhere. ${ }^{11,12,20}$

\section{Results and Discussions}

Detection of RCA in Adenoviral Products. To investigate contamination of RCA in rAdLacZ, PCR was performed using E1 specific primer. A 170-bp DNA fragment spann-

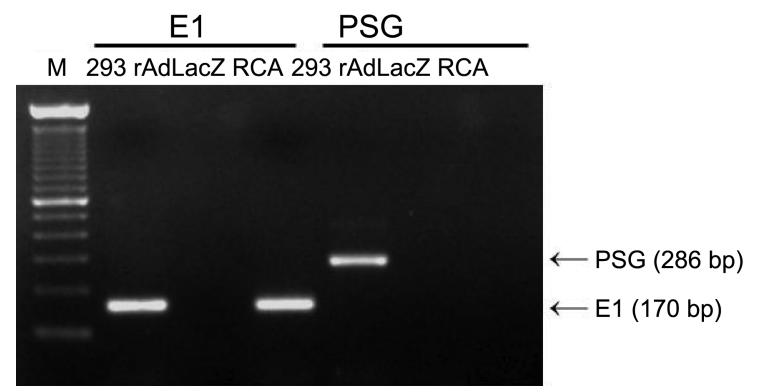

Figure 1. Detection of E1 (286-bp) DNA in adenoviral vectors and pregnancy-specific glycoprotein (PSG, 170-bp) in HEK 293 cells. M; 100 bp ladder marker.

(a)

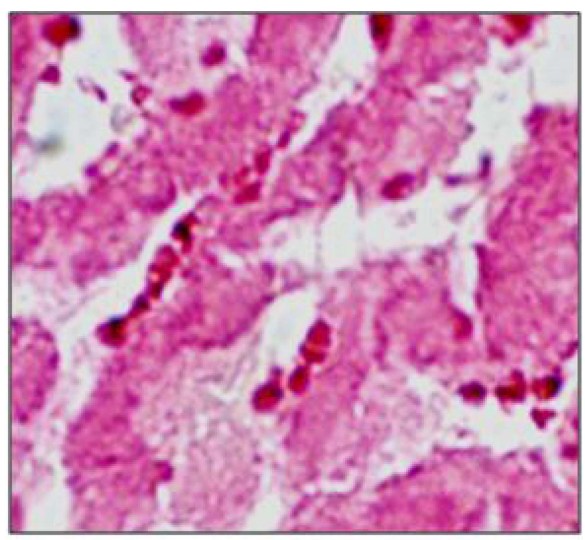

(b)

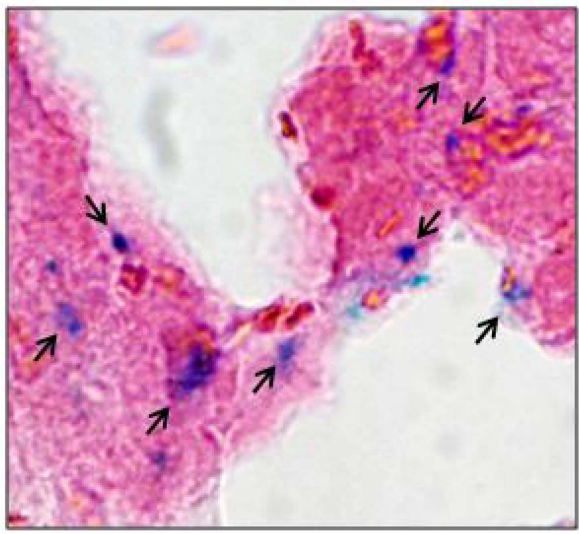

Figure 2. Histochemical staining of livers from rats 3 days after infection with $1 \times 10^{9}$ pfu of rAdLacZ in $0.1 \mathrm{~mL}$ of PBS. (a) Control rat was injected with only PBS. (b) rAdLacZ-infected rats. Arrows indicate $\beta$-gal positive staining. ing portions of the E1 region were detected in 293 cells and RCA, but not in rAdLacZ (Fig. 1). In addition, to exclude the possibility of the presence of 293 cell-derived E1 DNA in adenovirus products, we investigated a 286-bp DNA fragment of 293 cell-derived PSG genes. PSG DNA was only detected in 293 cells (Fig. 1). These findings suggest that there is no RCA contamination in purified rAdLacZ. We also performed a cytopathic effect (CPE) assay using A549 cells (human alveolar basal epithelial cells) and confirmed that there was no contamination in $\mathrm{AdLacZ}$ products (data not shown), this result was consistent with our previous report. ${ }^{11}$ For evidence of adenovirus mediated gene transfer, we checked the expression of $\beta$-gal at $72 \mathrm{~h}$ after exposure to rAdLacZ. Liver tissues were fixed and stained with the $\beta$-gal substrate, X-gal (5-bromo-4-chloro-3-indolyl- $\beta$-D-galactoside) because this virus has liver tropism leading to extensive hepatocellular transduction. ${ }^{21}$ Three days after injection, we analyzed reporter gene expression with liver pieces of rAdLacZ-infected rats. Blue staining was observed in rAdLacZ infected rats, but not in control (Fig. 2).

Plasma AA Levels in Adenovirus Infection Groups and Star Pattern Recognition. To analyze AA variation in adenovirus infected rats, we injected approximately $1 \times 10^{10}$ pfu of rAdLacZ and RCA to rats through a tail vein. Rats were sacrificed and individual plasma was collected for determination of AAs in three days following injection.

SIM chromatograms of AAs in rat plasma samples from control (Fig. 3(a)) rAdLacZ (Fig. 3(b)) and RCA (Fig. 3(c)) groups were shown in Figure 3. In all plasma samples, 16 AAs were simultaneously screened in a single run.

Alterations in 16 AA levels from 10 rat plasma samples were observed, with large variations within each group (Table 1). According to the Student's t-tests, in the rAdLacZ group, compared with the normal group, the level of aspartic acid $(P<0.009)$ was significantly elevated, whereas glycine $(P<0.001)$, valine $(P<0.03)$, threonine $(P<0.002)$, serine $(P<0.04)$, hydroxyproline $(P<0.05)$, glutamic acid $(P<$ $0.001)$, ornithine $(P<0.04)$, and glutamine $(P<0.04)$ were significantly decreased.

In the RCA group, compared with the normal group, levels of the following four AAs, valine $(P<0.01)$, leucine $(P<$ $0.005)$, and isoleucine $(P<0.01)$ as branched-chain amino acids (BCAAs), and proline $(P<0.004)$ were significantly elevated, while glycine $(P<0.001)$, threonine $(P<0.03)$, and glutamic acid $(P<0.005)$ were significantly reduced. These findings may explain the abnormal state of the adenovirus infected groups compared with the normal group. The rAdLacZ group, as compared with the RCA group, showed significantly different levels of the nine AAs, including glycine.

In this result, AA metabolism in normal and adenoviral infection groups showed significantly different patterns. This finding may explain for abnormal states, physiological events and synthesis of different proteins. Especially, altered AA metabolism of two adenoviral infection groups may explain for different infection types.

When AA levels from plasma samples of the rAdLacZ 


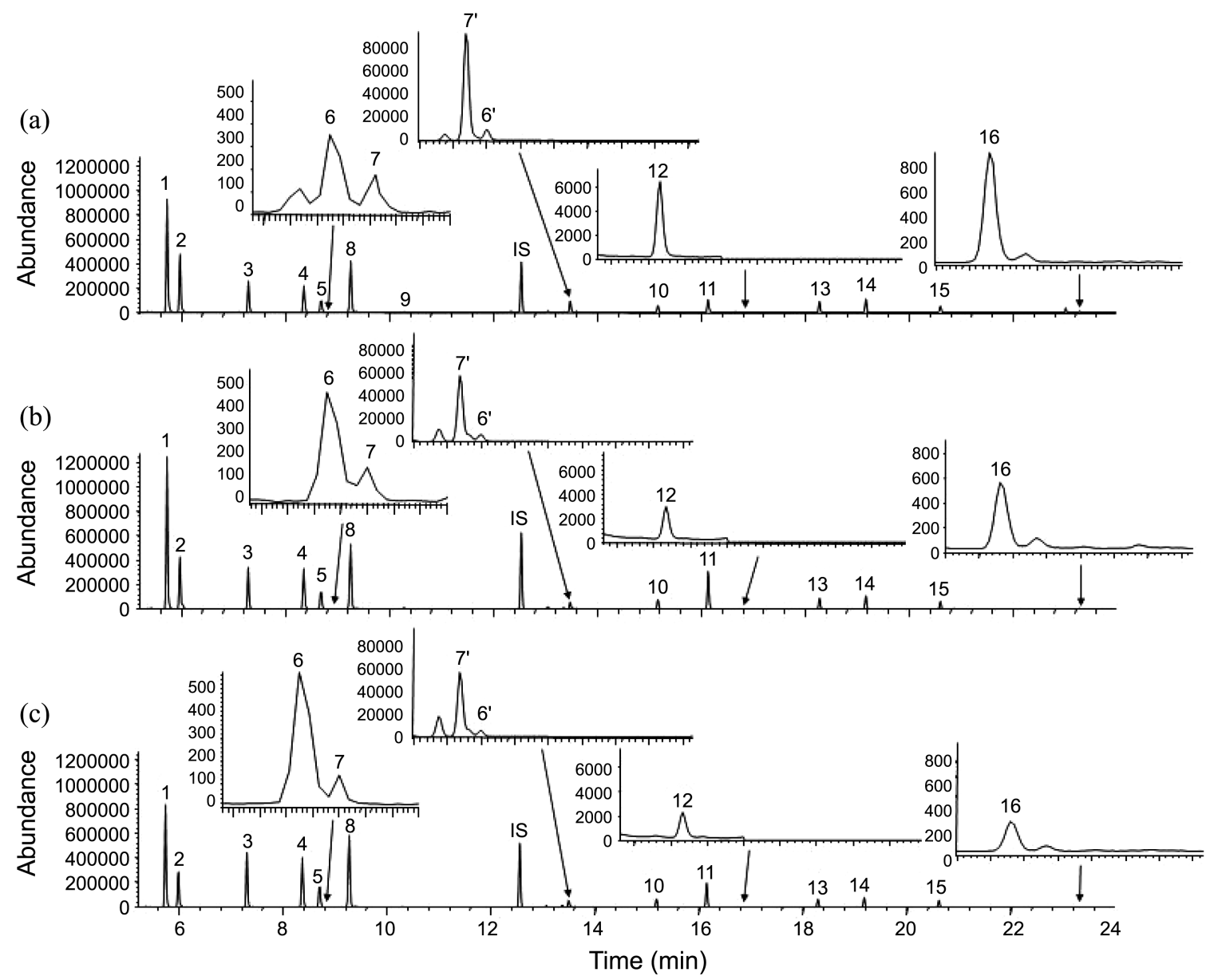

Figure 3. SIM chromatograms of amino acids in rat plasma from control (a), rAdLacZ (b) and RCA groups (c). 1, alanine; 2, glycine; 3, valine; $\mathbf{4}$, leucine; $\mathbf{5}$, isoleucine; $\mathbf{6}$, threonine as mono-EOC/mono-TBDMS derivatives; $\mathbf{7}$, serine as mono-EOC/mono-TBDMS derivatives; 8, proline; 9, pipecolic acid; 7', serine as mono-EOC/di-TBDMS derivatives; 6', threonine as mono-EOC/di-TBDMS derivatives; 10, phenylalanine; 11, aspartic acid; 12, hydroxyproline; 13, glutamic acid; 14, ornithine; 15, glutamine; 16, tyrosine. IS, 3,4-dimethoxybenzoic acid.

and RCA infection groups were normalized to corresponding control mean values, they were more informative by expressing elevation or depletion of AA levels in multiples (ranging from 0.49 to 1.48 ) of the control mean values (Table $1)$. When these normalized values were used as variables to draw star graphs that were hexadecagonal in shape and composed of 16 rays, differences in mean values among the control, rAdLacZ, and RCA groups were expressed as visual star patterns (Fig. 2).

In the star patterns of the two adenovirus infected groups, the aspartic acid level (ray 11) was higher in the rAdLacZ group (Fig. 4(a)), while the levels of valine (ray 3), leucine (ray 4), and isoleucine (ray 5), as BCAAs, and aspartic acid (ray 11) in the RCA group (Fig. 4(b)) were higher than those of the control group. However, the overall star patterns of other AAs including glutamic acid (ray 13) in both the rAdLacZ and RCA were smaller than those of the control group. As we expected, the size of RCA group was larger than that of the rAdLacZ group (Fig. 4(c)). This finding proves that RCA may lead to a remarkable metabolic confusion of AAs, including BCAAs, as compared with rAdLacZ, and glutamic acid was reduced more than $25 \%$ in the
rAdLacZ (Fig. 4(a)) and RCA (Fig. 4(b)) groups, as compared with the normal group, whereas aspartic acid was elevated more than $22 \%$ in both the rAdLacZ and RCA groups. This is the first demonstration for altered plasma free AA patterns in rAdLacZ and RCA groups as compare with the normal group.

In addition, the star patterns of the two infection groups were readily distinguishable and characteristic of each group.

As we hypothesized, the pattern of the RCA group was not similar to that of rAdLacZ in the star symbol plots (Fig. 4), and the BCAA level in the RCA group was larger than that of the rAdLacZ group. This may explain why RCA showed more distorted hexadecagonal forms for infected groups, as compared with the patterns of the rAdLacZ group, since the direct toxicity of RCA is able to cause replication, spreading, and transmission and can also induce inflammation by activation of inflammatory gene expression in the host. ${ }^{9,22,23}$ As shown in Table 1 and star symbol plots (Fig. 4), alteration of BCAAs between rAdLacZ and RCA infection groups was also observed. This result suggests that RCA can be a stimulation effecter of the immune system and a disturbance factor in immune metabolism by elevation of BCAA levels, 
Table 1. Amino acid levels in plasma of rats injected with rAdLacZ or RCA

\begin{tabular}{|c|c|c|c|c|c|c|c|}
\hline \multirow{2}{*}{ No. } & \multirow{2}{*}{ Amino acid } & \multicolumn{4}{|c|}{ Amino acid levels value $(\mu \mathrm{mol} / \mathrm{L}$, mean \pm S.D. $)$} & \multicolumn{2}{|c|}{ Normalized average values ${ }^{d}$} \\
\hline & & Control $(n=3)$ & rAdLacZ $(n=4)$ & $\operatorname{RCA}(n=3)$ & $P$-value ${ }^{c}$ & rAdLacZ & RCA \\
\hline 1 & Alanine & $362.7 \pm 56.6$ & $307.2 \pm 32.1(P<0.08)^{a}$ & $302.5 \pm 17.3(P<0.08)^{b}$ & $<0.4$ & 0.85 & 0.83 \\
\hline 2 & Glycine & $235.7 \pm 20.1$ & $177.7 \pm 7.1(P<0.001)$ & $154.6 \pm 5.5(P<0.001)$ & $<0.003$ & 0.75 & 0.66 \\
\hline 3 & Valine & $173.8 \pm 25.8$ & $141.6 \pm 6.4(P<0.03)$ & $228.7 \pm 5.3(P<0.01)$ & $<0.000004$ & 0.81 & 1.32 \\
\hline 4 & Leucine & $149.4 \pm 21.4$ & $137.5 \pm 6.8(P<0.2)$ & $208.8 \pm 4.9(P<0.005)$ & $<0.00001$ & 0.92 & 1.40 \\
\hline 5 & Isoleucine & $87.2 \pm 12.1$ & $80.1 \pm 2.6(P<0.1)$ & $113.4 \pm 2.1(P<0.01)$ & $<0.000005$ & 0.92 & 1.30 \\
\hline 6 & Threonine $^{e}$ & $201.7 \pm 5.4$ & $180.1 \pm 5.4(P<0.002)$ & $192.9 \pm 1.8(P<0.03)$ & $<0.006$ & 0.89 & 0.96 \\
\hline 7 & Serine $^{e}$ & $182.4 \pm 26.9$ & $140.2 \pm 25.2(P<0.04)$ & $151.4 \pm 3.6(P<0.06)$ & $<0.2$ & 0.77 & 0.83 \\
\hline 8 & Proline & $124.6 \pm 6.9$ & $116.2 \pm 4.7(P<0.06)$ & $148.2 \pm 4.3(P<0.004)$ & $<0.0001$ & 0.93 & 1.19 \\
\hline 9 & Pipecolic & $2.1 \pm 0.1$ & $2.1 \pm<0.1(P<0.2)$ & $2.0 \pm<0.1(P<0.1)$ & $<0.00001$ & 1.02 & 0.98 \\
\hline 10 & Phenylalanine & $36.6 \pm 3.5$ & $36.7 \pm 0.1(P<0.5)$ & $37.6 \pm 1.0(P<0.3)$ & $<0.07$ & 1.00 & 1.03 \\
\hline 11 & Aspartic acid & $57.5 \pm 15.8$ & $85.3 \pm 4.8(P<0.009)$ & $70.3 \pm 2.4(P<0.1)$ & $<0.002$ & 1.48 & 1.22 \\
\hline 12 & Hydroxyproline & $40.7 \pm 18.7$ & $21.4 \pm 6.7(P<0.05)$ & $20.1 \pm 1.7(P<0.07)$ & $<0.4$ & 0.53 & 0.49 \\
\hline 13 & Glutamic acid & $196.6 \pm 16.3$ & $142.9 \pm 9.7(P<0.001)$ & $146.5 \pm 8.6(P<0.005)$ & $<0.3$ & 0.73 & 0.75 \\
\hline 14 & Ornithine & $42.6 \pm 15.2$ & $25.9 \pm 1.4(P<0.04)$ & $24.8 \pm 1.1(P<0.06)$ & $<0.2$ & 0.61 & 0.58 \\
\hline 15 & Glutamine & $610.3 \pm 56.0$ & $507.2 \pm 64.7(P<0.04)$ & $577.3 \pm 71.5(P<0.3)$ & $<0.1$ & 0.83 & 0.95 \\
\hline 16 & Tyrosine & $73.0 \pm 29.3$ & $65.4 \pm 9.2(P<0.3)$ & $48.2 \pm 6.2(P<0.1)$ & $<0.02$ & 0.90 & 0.66 \\
\hline
\end{tabular}

${ }^{a}$ Student's t-test at $95 \%$ confidence level on the means of normal and group infected with rAdLacZ. ${ }^{b}$ Student's t-test at $95 \%$ confidence level on the means of normal and group infected with RCA. ${ }^{c}$ Student's t-test at $95 \%$ confidence level on the means of two groups infected with rAdLacZ and RCA. ${ }^{d}$ Values normalized to the corresponding control. ${ }^{e}$ Threonine and serine were calculated on sum of mono-EOC/mono-TBDMS and mono-EOC/diTBDMS derivatives.
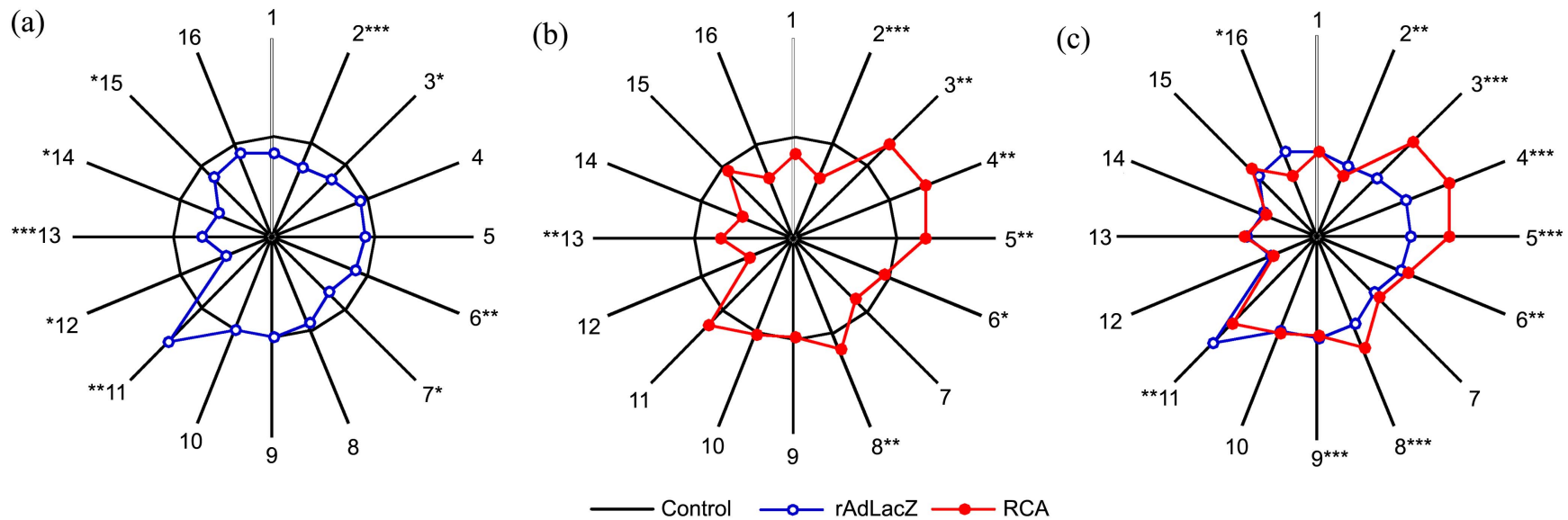

Figure 4. Star symbol plots of rAdLacZ, RCA, and control groups based on the mean levels of the 16 AAs variables after normalization to corresponding control mean values. (a) control $v s$. rAdLacZ, (b) control $v s$. RCA, and (c) rAdLacZ $v s$. RCA. $* P<0.05, * * P<0.01, * * * P$ $<0.001$; Student's $t$-test at $95 \%$ confidence level. Numbers on the rays correspond to those in Table 1 .

and RCA has direct toxicity in vivo compared with rAdLacZ.

Resulting hexadecagonal star patterns between the rAdLacZ and RCA groups were deformed and characteristic of each group (Fig. 4(c)). Generally, the aspartic acid and glutamic acid could be the result of transamination by aspartate aminotransferase (AspAT), which is known as a pyridoxal5'-phosphate (PLP) dependent enzyme that catalyzes reversible transamination between the $\mathrm{C} 4$ and $\mathrm{C} 5$ dicarboxylic substrates. $^{24,25}$ Thus, this result may explain for metabolic disturbance from transamination by AspAT. And the star symbol plotting method may be a useful application for visual monitoring and screening of adenoviral infection states.

In addition, adenoviral infection induces a host cell response by post-translational modification of cellular proteins. ${ }^{26-28}$ In particular, arginine methylation during viral infection is apparently related with transcriptional regulation, protein localization, signal transduction, and protein-protein interactions that change gene expression. More in depth modified AA studies are needed to monitor modified metabolic changes, e.g., methylated AAs of adenovirus introduction in vivo due to the importance of their safe use.

The present method is expected to be useful for visual monitoring of AAs as biochemical indicators for infection with adenoviral vector or RCA, which is an important issue for successful treatment in adenoviral vector-based gene therapy. Thus, the present methods may be useful for understanding changes in AAs caused by infection with recombinant adenovirus or RCA and for reducing a variety of safety 
issues in human gene therapy trials.

\section{Conclusion}

Changes in plasma free AA patterns in rAdLacZ and RCA groups, compared with the control group, may explain viral infection-related alterations in AA metabolism. In addition, the star patterns of the two infection groups were readily distinguishable and characteristic of each group; the star symbol plotting method may be a useful application for visual monitoring and screening of adenoviral infection states.

Acknowledgments. This work was supported by the Korea Research Foundation (KRF) grant funded by the Basic Science Research Program through the National Research Foundation of Korea funded by the Ministry of Education, Science, and Technology (2010-0023846) to G. Lee, Priority Research Centers Program through the National Research Foundation of Korea (NRF) funded by the Ministry of Education, Science and Technology (2010-0028294), and by Mid-career Researcher Program through NRF grant funded by the MEST (No. 2009-0084444) to D. W. Kim.

\section{References}

1. Baum, B. J.; Goldsmith, C. M.; Kok, M. R.; Lodde, B. M.; van Mello, N. M.; Voutetakis, A.; Wang, J.; Yamano, S.; Zheng, C. Immunol. Lett. 2003, 90, 145.

2. Campochiaro, P. A.; Nguyen, Q. D.; Shah, S. M.; Klein, M. L.; Holz, E.; Frank, R. N.; Saperstein, D. A.; Gupta, A.; Stout, J. T.; Macko, J.; DiBartolomeo, R. Hum. Gene. Ther. 2006, 17, 167.

3. Griesenbach, U.; Geddes, D. M.; Alton, E. W. Gene. Ther. 2006, 13, 1061 .

4. Jaras, M.; Brun, A. C.; Karlsson, S.; Fan, X. Exp. Hematol. 2007, 35,343 .

5. Kawabata, K.; Sakurai, F.; Yamaguchi, T.; Hayakawa, T.; Mizuguchi, H. Mol. Ther. 2005, 12, 547.
6. Roth, J. A. Expert. Opin. Biol. Ther. 2006, 6, 55.

7. Graham, F. L.; Prevec, L. Mol. Biotechnol. 1995, 3, 207.

8. Ishii-Watabe, A.; Uchida, E.; Iwata, A.; Nagata, R.; Satoh, K.; Fan, K.; Murata, M.; Mizuguchi, H.; Kawasaki, N.; Kawanishi, T.; Yamaguchi, T.; Hayakawa, T. Mol. Ther. 2003, 8, 1009.

9. Liu, Q.; Muruve, D. A. Gene. Ther. 2003, 10, 935.

10. Lochmuller, H.; Jani, A.; Huard, J.; Prescott, S.; Simoneau, M.; Massie, B.; Karpati, G.; Acsadi, G. Hum. Gene. Ther. 1994, 5, 1485.

11. Paik, M. J.; Park, K. H.; Park, J. J.; Kim, K. R.; Ahn, Y. H.; Shin, G. T.; Lee, G. J. Biochem. Mol. Biol. 2007, 40, 119.

12. Paik, M. J.; Lee, K. A.; Park, C. S.; Ahn, Y. H.; Jeong, R.; Kim, K. R.; Lee, G. Clin. Chim. Acta 2007, 380, 228.

13. Moinard, C.; Cynober, L.; de Bandt, J. P. Eur. Clin. Nutr. 2005, 24 , 184.

14. Okun, J. G.; Kolker, S.; Schulze, A.; Kohlmuller, D.; Olgemoller, K.; Lindner, M.; Hoffmann, G. F.; Wanders, R. J.; Mayatepek, E. Biochimica. Et. Biophysica. Acta 2002, 1584, 91.

15. Olpin, S. E. P.L.E.F.A. 2004, 70, 293

16. Teti, D.; Visalli, M.; McNair, H. J. Chromatogr. 2002, 781, 107.

17. Maranga, L.; Aunins, J. G.; Zhou, W. Biotech. Bioengin. 2005, 90, 645.

18. Paik, M. J.; Moon, S. M.; Kim, K. R.; Choi, S.; Ahn, Y. H.; Lee, G. Biomed. Chromatogr. 2008, 22, 339.

19. Paik, M. J.; Cho, E. Y.; Kim, H.; Kim, K. R.; Choi, S.; Ahn, Y. H.; Lee, G. Biomed. Chromatogr. 2008, 22, 450.

20. Paik, M. J.; Li, W. Y.; Ahn, Y. H.; Lee, P. H.; Choi, S.; Kim, K. R.; Kim, Y. M.; Bang, O. Y.; Lee, G. Clin. Chim. Acta 2009, 402, 25.

21. Wood, M.; Perrotte, P.; Onishi, E.; Harper, M. E.; Dinney, C.; Pagliaro, L.; Wilson, D. R. Cancer. Gene. Ther. 1999, 6, 367.

22. Ghosh, S. S.; Gopinath, P.; Ramesh, A. Appl. Biochem. Biotechnol. 2006, 133,9 .

23. Smith, T. A.; Mehaffey, M. G.; Kayda, D. B.; Saunders, J. M.; Yei, S.; Trapnell, B. C.; McClelland, A.; Kaleko, M. Nat. Genet. 1993, $5,397$.

24. Islam, M. M.; Hayashi, H.; Kagamiyama, H. J. Biochem. 2003, 134, 277.

25. Kim, H.; Ikegami, K.; Nakaoka, M.; Yagi, M.; Shibata, H.; Sawa, Y. Biosci. Biotechnol. Biochem. 2003, 67, 490.

26. Kleinberger, T. Apoptosis 2000, 5, 211.

27. Branton, P. E.; Roopchand, D. E. Oncogene 2001, 20(54), 7855.

28. Kzhyshkowska, J.; Kremmer, E.; Hofmann, M.; Wolf, H.; Dobner, T. Biochem. J. 2004, 383, 259. 